\title{
Constrained potential method for false vacuum decays
}

\section{Jae-hyeon Park}

Deutsches Elektronen-Synchrotron DESY,

Notkestraße 85, 22607 Hamburg, Germany

E-mail: jae-hyeon.park@desy.de

Received December 20, 2010

Accepted February 11, 2011

Published February 21, 2011

Abstract. A procedure is reported for numerical analysis of false vacuum transition in a model with multiple scalar fields. It is a refined version of the approach by Konstandin and Huber. The alteration makes it possible to tackle a class of problems that was difficult or unsolvable with the original method, i.e. those with a distant or nonexistent true vacuum. An example with an unbounded-from-below direction is presented.

Keywords: cosmological phase transitions, cosmology of theories beyond the SM

ArXiv ePrint: 1011.4936 
This is a note on numerical computation of the decay rate of a false vacuum within a potential of multiple scalar fields. The aim is to describe a new strategy to obtain the bounce configuration, that is based on an earlier idea by Konstandin and Huber splitting the task into two stages [1]. The first is to search for an intermediate solution in one-dimensional spacetime using an improved potential. In the second, the field profile is stretched smoothly to that in four dimensions.

One advantage of the original approach is that the outcome, by construction, must be either a valid solution of the Euclidean equation of motion or something clearly wrong. For instance, this property is not shared by the improved action method [2]. The authors of ref. [3] use this method to find a fit and then check its quality by taking the kinetic to potential ratio within the action. This ratio must be -2 for a field configuration to leave the action stationary under an infinitesimal scale transformation $[4,5]$. Being a necessary condition, however, this is not more than a consistency check and one cannot be sure that the numerical data is indeed a bounce until it is put into the equation.

Its virtues notwithstanding, the original approach has drawbacks due to the use of an improved potential that render it suboptimal in some circumstances. Particularly problematic is a case in which the false and the true vacua are separated far apart. This can arise for instance in the Minimal Supersymmetric Standard Model (MSSM) with a large trilinear term unless it involves a top squark [6,7]. One can put an upper limit on such a term by demanding that the lifetime of the standard vacuum be longer than the age of the universe $[8,9]$. The same criterion has been employed in a study of maximal flavour violation by a soft trilinear coupling [10]. For this last work, a modification has been made to the original approach, which is to be the subject of this article.

Consider the potential depicted in figure 1, where it has been inverted to make it easy to imagine a classical motion. It is a function of scalar fields collectively denoted by $\phi$. The problem is to estimate the rate of transition from the local minimum $\phi_{+}$to the state $\phi_{-}$with the lowest energy. Note that the notations $\phi_{ \pm}$in this article follow ref. [11] and are reversed with respect to ref. [1].

Using a semiclassical approximation [11, 12], one finds that the decay rate of a false vacuum per unit volume is

$$
\Gamma / V=A \exp (-S[\bar{\phi}]),
$$

where the determinantal factor $A$ comes from the Gaussian functional integral around the stationary point $\bar{\phi}$, called the bounce, of the Euclidean action $S$. The formula for $A$ can be found in ref. [12]. It is very difficult to compute in practice and so can only be estimated to be around the characteristic mass scale of the problem. Normally, evaluation of $A$ is exponentially less important than that of $S[\bar{\phi}]$. Without loss of generality, the dominant bounce can be assumed to be $O(4)$-invariant [5]. Therefore, one can let $\phi$ be a function of the single coordinate $\rho \equiv \sqrt{\tau^{2}+\mathrm{x}^{2}}$, and write $S$ in the form,

$$
S[\phi(\rho)]=2 \pi^{2} \int_{0}^{\infty} d \rho \rho^{3}\left[\frac{1}{2}\left(\frac{d \phi}{d \rho}\right)^{2}+V(\phi)\right] .
$$

The ultimate goal is then to find the solution $\bar{\phi}$ of the Euler-Lagrange equation,

$$
\frac{d^{2} \phi}{d \rho^{2}}+\frac{3}{\rho} \frac{d \phi}{d \rho}=\nabla V(\phi)
$$




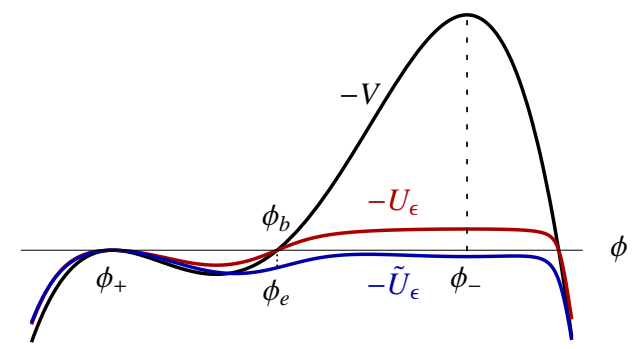

Figure 1. An example potential $V$ and the corresponding improved potentials $U_{\epsilon}$ and $\widetilde{U}_{\epsilon}$, displayed upside down. The false and the true vacua are designated by $\phi_{+}$and $\phi_{-}$, respectively. Between them, there are $\phi_{e}$ and $\phi_{b}$. The former denotes any point with $V\left(\phi_{e}\right)=V\left(\phi_{+}\right)$and the latter such a point on the bounce.

derived from the above Euclidean action, that meets the boundary conditions,

$$
\frac{d \phi}{d \rho}(\rho=0)=0, \quad \phi(\rho \rightarrow \infty)=\phi_{+} .
$$

The original approach [1] is based on the idea that one can generalise the equation of motion to that in $\alpha$ spacetime dimensions,

$$
\frac{d^{2} \phi}{d \rho^{2}}+\frac{\alpha-1}{\rho} \frac{d \phi}{d \rho}=\nabla V(\phi)
$$

which corresponds to the generalised action,

$$
\widetilde{S}_{\alpha}[\phi(\rho)]=\int_{0}^{\infty} d \rho \rho^{(\alpha-1)}\left[\frac{1}{2}\left(\frac{d \phi}{d \rho}\right)^{2}+V(\phi)\right] .
$$

The procedure can be divided into two parts: to solve the equation in the undamped case with $\alpha=1$ and then to deform the solution to its final shape. The deformation part has no problem and shall be presented later when the refined version is described.

The first part for the undamped case is summarised. If $\alpha=1$, the damping term in (5) disappears and so the motion conserves energy. Therefore, one can reproduce the initial condition in (4) in the limit where the starting and the ending points are degenerate. The degeneracy is approximated by flattening out part of the potential that is lower than the false vacuum. The solution is found by minimising the action (6) for $\alpha=1$. More specifically, the procedure is made up of the following steps.

1. Find the minimum of $\widetilde{S}_{1}$ with $V$ in (6) replaced by the improved potential,

$$
U_{\epsilon}(\phi)=\frac{V(\phi)-V\left(\phi_{+}\right)}{2}+\left[\frac{\left[V(\phi)-V\left(\phi_{+}\right)\right]^{2}}{4}+\epsilon^{2}\right]^{1 / 2},
$$

satisfying the boundary conditions,

$$
\phi(0)=\phi_{-}, \quad \phi(T)=\phi_{+} .
$$

The shape of $U_{\epsilon}$ is illustrated in figure 1 with $\epsilon$ set to $\left[V\left(\phi_{-}\right)-V\left(\phi_{+}\right)\right] / 10$. One can accelerate the convergence by making a further modification to $U_{\epsilon}$ to get

$$
\widetilde{U}_{\epsilon}(\phi)=U_{\epsilon}(\phi)+\Delta U_{\epsilon}(\phi)
$$


shown in figure 1, with a small perturbation,

$$
\Delta U_{\epsilon}(\phi)=-2 \epsilon \frac{\left|\phi-\phi_{+}\right|^{3}}{\left|\phi_{-}-\phi_{+}\right|^{3}}+3 \epsilon \frac{\left|\phi-\phi_{+}\right|^{2}}{\left|\phi_{-}-\phi_{+}\right|^{2}} .
$$

The initial choice of the parameter $\epsilon$ and the time $T$ may be

$$
\begin{aligned}
\epsilon_{\text {init }} & =V\left(\phi_{-}\right)-V\left(\phi_{+}\right), \\
T_{\text {init }} & =20\left|\phi_{-}-\phi_{+}\right| / \sqrt{8 V_{b}},
\end{aligned}
$$

where $V_{b}$ is the height of the potential barrier.

2. Find the point $\phi_{e}$ between $\phi_{-}$and $\phi_{+}$such that $V\left(\phi_{e}\right)=V\left(\phi_{+}\right)$within the configuration obtained in the previous step. Truncate the part before $\phi_{e}$, i.e. the configuration on the plateau.

3. Repeat minimisation with the new set of boundary conditions,

$$
\phi(0)=\phi_{e}, \quad \phi(T)=\phi_{+},
$$

while iteratively sending $\epsilon$ down to zero. Initially $\phi_{e}$ is set to the point found above and then it is allowed to move freely on the submanifold with $V\left(\phi_{e}\right)=V\left(\phi_{+}\right)$.

4. The last minimum for $\epsilon=0$ is the solution of the undamped equation,

$$
\frac{d^{2} \phi}{d \rho^{2}}=\nabla V(\phi)
$$

which is (5) for $\alpha=1$, and obeys the boundary conditions,

$$
\frac{d \phi}{d \rho}(0)=0, \quad \phi(T)=\phi_{+} .
$$

There are a couple of points of concern. First, the improved potential (7), sketched in figure 1, has a plateau on which the solution of step 1 has to spend a long time. In fact this is what the improved potential is designed for so that the initial condition in (4) can be approximated. As already stated in ref. [1], however, this costs generically many lattice points to store a long part of the path that is to be discarded eventually. This grows more and more problematic as $\phi_{-}$moves away from $\phi_{b}$, and hampers the computation. Second, step 3 might lead to a numerical instability. The intention of this step is to find a path that starts from $\phi_{e}$ and then rolls down the inverted potential. Although modified to have a plateau, the improved potential still has a part with $U_{\epsilon}(\phi)<U_{\epsilon}\left(\phi_{e}\right)=U_{\epsilon}\left(\phi_{+}\right)$for a finite $\epsilon$. After starting from $\phi_{e}$, the path may prefer to climb up the inverted potential and spend time in this region to minimise the action. This can be avoided by using $\widetilde{U}_{\epsilon}$ instead of $U_{\epsilon}$. With a judicious choice of $\Delta U_{\epsilon}$, one can make $\phi_{+}$the global minimum of $\widetilde{U}_{\epsilon}$ so that the system tries to spend as much time as possible around $\phi_{+}$at the end of the path. Note that for this, $\epsilon$ in (10) should be different from $\epsilon$ in (7) in general. The initial condition in (15) is violated by $\widetilde{U}_{\epsilon}\left(\phi_{e}\right)-V\left(\phi_{e}\right)$, which eventually goes away as $\epsilon \rightarrow 0$.

The first point makes it difficult to analyse many interesting problems. For instance, vacuum transition triggered by the stau trilinear coupling in the MSSM is studied in ref. [9]. 
The charge-breaking global minimum in the scalar potential is far from the local one due to the small tau Yukawa coupling. To overcome this problem, they first found the bounce of a temporary potential with two nearly degenerate minima using the above steps, and then made a continuation to the actual potential by iteration. In the course of deforming the potential, one should be careful not to introduce a singular behaviour.

In what follows, a more streamlined procedure shall be presented. It does not cost extra lattice points to be truncated in the end. It does not need a deformation of the potential. It does not rely on the location of the true vacuum. Consequently, it can deal with a potential that has widely separated local and global minima or that is even unbounded from below.

The main idea is to exploit the energy conservation in the undamped case, which was already mentioned in ref. [1]. This feature enables one to replace the Neumann boundary condition of the original problem by a constraint on the potential. As pointed out above, fixing the potential at $\rho=0$ is not enough to have the desired solution be a minimum since a path can lower the action further by shooting up the inverted potential and staying in a region with $V(\phi)<V\left(\phi_{+}\right)$. The refinement here is to eliminate those paths by additional constraints.

Including the preparation and the continuation stages as well, the new series of steps would be as follows.

1. Find a point $\phi_{e}$ such that $V\left(\phi_{e}\right)=V\left(\phi_{+}\right)$that is on the other side of the barrier. For instance, one can walk over the barrier along a valley starting from $\phi_{+}$until the level comes down back to $V\left(\phi_{+}\right)$.

2. Construct an initial configuration $\phi(\rho)$ such that

$$
\phi(0)=\phi_{e}, \quad \phi(T)=\phi_{+} .
$$

The simplest choice would be a step-like profile in which $\phi(\rho)$ is constant except for one jump somewhere in the middle, possibly around $\rho=0.1 T$.

Choosing a suitable $T$ is important for efficient evaluation. Recall that (17a) can substitute for (15) only in the limit of energy conservation. Realising this limit would require infinite $T$, whereas a small $T$ saves the computation time and storage. However, it is difficult to estimate an optimal $T$ before doing any minimisation. Initially, one may try (12) with $\phi_{e}$ instead of $\phi_{-}$and $V_{b}$ from the previous step, and then $T$ can be adjusted later as explained below.

Optionally to prepare a better initial profile, one could perform a single-field minimisation of $\widetilde{S}_{1}$ using as the field the position on the segment connecting $\phi_{+}$and $\phi_{e}$. One should repeat this process until $T$ is adjusted appropriately. The criterion for this and the constraints on the potential are the same as in the next step.

3. Find a minimum of $\widetilde{S}_{1}$ obeying the boundary conditions,

$$
V(\phi(0))=V\left(\phi_{+}\right), \quad \phi(T)=\phi_{+},
$$

in combination with

$$
V(\phi(\rho)) \geq V\left(\phi_{+}\right) \quad \text { for } \quad 0<\rho<T .
$$

The inequality (17b) should be enforced at every lattice point in order to prevent the unwanted 'upshooting'. 
One should repeat the minimisation with an increased $T$ until energy is sufficiently conserved. It usually works to require that the average difference between the kinetic and the potential energy densities be less than around $1 \%$ of the barrier height measured within the resulting profile.

4. The minimum is a solution of the equation,

$$
\frac{d^{2} \phi}{d \rho^{2}}+\frac{\alpha-1}{\rho+\Delta \rho} \frac{d \phi}{d \rho}=\nabla V(\phi)
$$

with the damping term killed by $\alpha=1$, that complies with the boundary conditions (15). In comparison to (5), there is an additional offset parameter $\Delta \rho$ that has been introduced to avoid a pathological behaviour near to $\rho=0[1]$.

5. Make a continuation to the damped case by gradually increasing $\alpha$ from 1 to 4 with $\Delta \rho$ fixed around $0.75 T$, and then send $\Delta \rho$ also to zero. One can choose to stop at $\alpha=3$ for tunnelling in a finite temperature system [13]. For each pair of $\alpha$ and $\Delta \rho$, one can linearise (18) using the series expansion of the right hand side,

$$
\nabla V(\phi) \approx \nabla V(\widetilde{\phi})+(\phi-\widetilde{\phi}) \cdot \nabla \nabla V(\widetilde{\phi}),
$$

and then iteratively solve it by matrix inversion. Boundary conditions are set by (15).

6. The final solution is the bounce configuration.

It should be reminded that steps 4 and 5 have been copied from ref. [1]. Modifications have been made only to the steps for the undamped case.

The proof of step 3 follows directly from that of step 1 in the original method [1]. First of all, consider a solution determined by the improved potential (7) and the boundary conditions (8). It is a minimum of the action since $\phi_{-}$is the global minimum of $U_{\epsilon}$. Next, take the limit of $\epsilon \rightarrow 0$. Since the path on the plateau makes no contribution to the action, the rest of the path becomes a minimum of $\widetilde{S}_{1}$ subject to the constraints (17). This motion off the plateau satisfies the equation (14) and the boundary conditions (15) which are equivalent to (17a) by virtue of energy conservation. Note that the improved potential is invoked only for the proof but never appears in the practical numerical calculation.

There is a subtlety regarding the bounce as a minimum of the action under the conditions (17). In the limit of $T \rightarrow \infty$, there is a flat direction of minima that initially spend different amounts of time staying at $\phi_{e}$ before rolling down. Any of this family of minima qualifies in principle as a solution of the undamped equation. In practice, however, it is desirable to find a path that does not have a long constant part to save computation resources. This is why the initial profile in step 2 was set to have a jump at a position close to $\rho=0$. For a finite $T$, the flat direction is slightly lifted. The minimum search routine should be able to deal with this kind of continuously (quasi-)degenerate minima. Also, the termination condition should be tuned in such a way that the routine stops after reaching a point that is acceptable to step 4 even though it is not the exact minimum. This needs some experiments.

The minimisation problem in step 3 is a nontrivial task termed nonlinear programming [14] which is a broad research area on its own. One possible strategy is the following (see e.g. [15]). An equality constraint is taken care of by employing a Lagrange multiplier. An inequality constraint is replaced with an equality by introducing a slack variable with a limited range. This limit is fulfilled by adding a barrier term to the function to be minimised. 
This term is a product of the barrier parameter $\mu$ and a barrier function of the slack variable. The solution of the original problem is obtained by solving a sequence of subproblems with $\mu$ successively decreasing towards zero. At each 'outer iteration', the subproblem with the fixed $\mu$ is solved by a damped Newton's method which involves 'inner iterations', and then this and the previous outcomes are used for making the initial guess for the next subproblem with a smaller $\mu$. The method in ref. [15] needs the 'outer loop' to be repeated several times. If the algorithm is too hard to implement, one can let a library do the job. For instance, the example exhibited below uses the Ipopt package [16].

It might be of interest to compare this and the original approaches for a problem that both can solve. Suppose that one introduces $n$ lattice points each holding $f$ unfixed field variables between $\phi_{+}$and $\phi_{e}$ (see figure 1). The constrained minimisation problem in step 3 roughly corresponds to applying Newton's method to a function of $n(f+2)-1$ free variables, i.e. $n f$ field values, $n$ Lagrange multipliers, and $n-1$ slack variables. This procedure is repeated, each time with a decreased barrier parameter. To use the original approach on the other hand, one needs additional $N$ points from $\phi_{e}$ to $\phi_{-}$except $\phi_{e}$ that has already been counted. The function to be minimised depends on $(n+N) f$ variables in the first step and $n f+1$ in the second including one Lagrange multiplier. Newton's method is repeated while iteratively driving $\epsilon \rightarrow 0$. Given only this information, it is difficult to tell which way is more efficient on general grounds. Nevertheless, it is obvious that a case with large $N$ is better handled with the new strategy since each Newton iteration becomes very expensive with the original. This happens when $\phi_{-}$is distant from $\phi_{e}$. Another limit arises with large $f$ that may favour the new approach over the original. In this case, the number of auxiliary degrees of freedom $2 n-1$ in the former becomes much smaller than $N f$ in the latter.

Now, the refined procedure is applied to a problem with two real fields as a demonstration. The scalar potential is chosen to be

$$
V=\frac{m_{1}^{2}}{2} \phi_{1}^{2}+\frac{m_{2}^{2}}{2} \phi_{2}^{2}-\frac{A}{2} \phi_{1} \phi_{2}^{2}+g^{2}\left(\phi_{1}^{2}-\phi_{2}^{2}\right)^{2} .
$$

This potential is unbounded from below. In this sense, it is qualitatively different from the examples in ref. [1]. Clearly, one cannot impose the boundary conditions (8) since there exists no $\phi_{-}$. Therefore, the original method is not applicable unless the potential is altered. There is a minimum with $V=0$ at $\phi_{1}=\phi_{2}=0$ that is local as long as $A \neq 0$. One can see the metastability easily by taking the field direction, $\phi_{1}=\phi_{2}=a$, that cuts out the quartic term leaving

$$
V_{\text {flat }}=\frac{1}{2}\left(m_{1}^{2}+m_{2}^{2}\right) a^{2}-\frac{A}{2} a^{3} .
$$

Along this direction, a barrier starts from the origin and ends at the position where $A a=$ $m_{1}^{2}+m_{2}^{2}$, after which the potential drops below zero and keeps falling down.

The shape of the potential is illustrated in figure 2. The masses and couplings in (20) are set to be

$$
m_{1}^{2}=3.0, \quad m_{2}^{2}=0.5, \quad A=1.5, \quad g=0.4 .
$$

There is a force that drives the path off the straight directions with $\phi_{1}= \pm \phi_{2}$, arising from the gradients of both the trilinear and the mass terms. A large difference between $m_{1}^{2}$ and $m_{2}^{2}$ has been introduced to emphasise this dynamics.

The white contour corresponds to the point $\phi_{e}$ in figure 1 . Finding any point on the curve is enough to complete step 1. Successful execution up to step 3 should lead to the undamped solution for $\alpha=1$ shown in figure 2. Notice that the starting point of the 


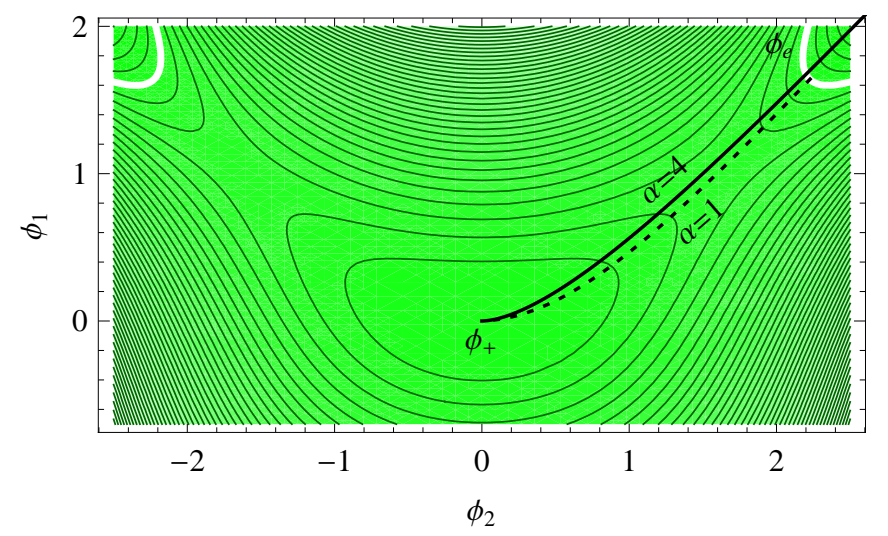

Figure 2. Contours of the scalar potential (20). The local minimum is marked with $\phi_{+}$. The white curve is the set of points $\phi_{e}$ at the same level as $\phi_{+}$. The dotted and the solid curves show the bounces in the undamped and the damped cases, respectively.

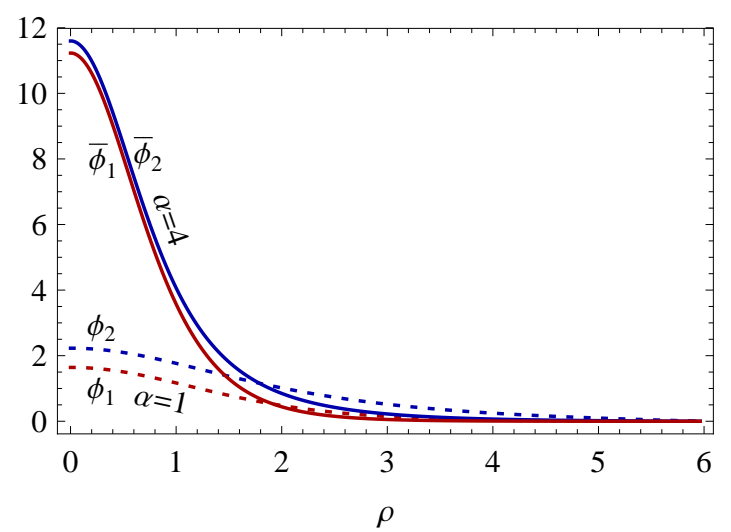

Figure 3. Field profiles of the bounces. The dotted and the solid curves show the undamped and the damped cases, respectively.

trajectory is on the white curve as enforced by (17a). Then one can proceed to take steps 4 and 5 to deform this path to the final bounce for $\alpha=4, \Delta \rho=0$. It is also partly visible in the same figure.

The solutions are plotted as functions of the time in figure 3. As $\alpha$ increases, the starting point retreats from the false vacuum due to the damping term in (18). Notice that the path does not diverge indefinitely even with an unbounded-from-below potential. This is understandable from the fact that the original problem formulated in (3) and (4) has nothing to do with any information on the global minimum. For the same reason, the procedure described in this article does not need to explore deep into the potential for an unreachable bottom. One can put this solution back into the Euclidean action (2) to calculate the final answer,

$$
S[\bar{\phi}]=488 .
$$

In this problem, the potential has a symmetry with respect to the reflection $\phi_{2} \rightarrow-\phi_{2}$. This pairs a bounce with the other that makes exactly the same contribution to the decay rate. Therefore, one must multiply the decay rate (1) by 2 .

It is straightforward to write the latticised versions of the fields, the Euclidean action, the Euler-Lagrange equation, and the boundary conditions, and they can be found in ref. [1] 
for instance. The same reference reports an analysis of discretisation error. It is determined by the final iteration step for continuation to four dimensions that is common to the present and the original methods.

\section{Acknowledgments}

The author thanks Ahmed Ali for helpful comments.

\section{References}

[1] T. Konstandin and S.J. Huber, Numerical approach to multi dimensional phase transitions, JCAP 06 (2006) 021 [hep-ph/0603081] [SPIRES].

[2] A. Kusenko, Improved action method for analyzing tunneling in quantum field theory, Phys. Lett. B 358 (1995) 51 [hep-ph/9504418] [SPIRES];

[3] A. Kusenko, P. Langacker and G. Segre, Phase Transitions and Vacuum Tunneling Into Charge and Color Breaking Minima in the MSSM, Phys. Rev. D 54 (1996) 5824 [hep-ph/9602414] [SPIRES].

[4] G.H. Derrick, Comments on nonlinear wave equations as models for elementary particles, J. Math. Phys. 5 (1964) 1252 [SPIRES].

[5] S.R. Coleman, V. Glaser and A. Martin, Action Minima Among Solutions to a Class of Euclidean Scalar Field Equations, Commun. Math. Phys. 58 (1978) 211 [SPIRES].

[6] J.A. Casas, A. Lleyda and C. Muñoz, Strong constraints on the parameter space of the MSSM from charge and color breaking minima, Nucl. Phys. B 471 (1996) 3 [hep-ph/9507294] [SPIRES].

[7] J.A. Casas and S. Dimopoulos, Stability bounds on flavor-violating trilinear soft terms in the MSSM, Phys. Lett. B 387 (1996) 107 [hep-ph/9606237] [SPIRES].

[8] M. Endo, K. Hamaguchi and K. Nakaji, Probing High Reheating Temperature Scenarios at the LHC with Long-Lived Staus, JHEP 11 (2010) 004 [arXiv: 1008.2307] [SPIRES].

[9] J. Hisano and S. Sugiyama, Charge-breaking constraints on left-right mixing of stau's, Phys. Lett. B 696 (2011) 92 [arXiv: 1011.0260] [SPIRES].

[10] J.-h. Park, Metastability bounds on flavour-violating trilinear soft terms in the MSSM, arXiv: 1011.4939 [SPIRES].

[11] S.R. Coleman, The Fate of the False Vacuum. 1. Semiclassical Theory, Phys. Rev. D 15 (1977) 2929 [SPIRES].

[12] C.G. Callan Jr. and S.R. Coleman, The Fate of the False Vacuum. 2. First Quantum Corrections, Phys. Rev. D 16 (1977) 1762 [SPIRES].

[13] A.D. Linde, On the Vacuum Instability and the Higgs Meson Mass, Phys. Lett. B 70 (1977) 306 [SPIRES];

A.D. Linde, Fate of the False Vacuum at Finite Temperature: Theory and Applications, Phys. Lett. B 100 (1981) 37 [SPIRES];

A.D. Linde, Decay of the False Vacuum at Finite Temperature, Nucl. Phys. B 216 (1983) 421 [SPIRES];

I. Affleck, Quantum Statistical Metastability, Phys. Rev. Lett. 46 (1981) 388 [SPIRES].

[14] http://wiki.mcs.anl.gov/NEOS/index.php/Nonlinear_Programming_FAQ.

[15] A. Wächter and L.T. Biegler, On the Implementation of a Primal-Dual Interior Point Filter Line Search Algorithm for Large-Scale Nonlinear Programming, Math. Program. 106 (2006) 25.

[16] https://projects.coin-or.org/Ipopt. 